\title{
neoSYCL: a SYCL implementation for SX-Aurora TSUBASA
}

\author{
Yinan Ke \\ keyinan@hpc.is.tohoku.ac.jp \\ Graduate School of Information \\ Sciences, Tohoku University
}

\author{
Mulya Agung \\ agung@tohoku.ac.jp \\ Cyberscience Center, Tohoku \\ University
}

\author{
Hiroyuki Takizawa \\ takizawa@tohoku.ac.jp \\ Cyberscience Center, Tohoku \\ University
}

\begin{abstract}
Recently, the high-performance computing world has moved to more heterogeneous architectures. Thus, it has become a standard practice to offload a part of application execution to dedicated accelerators. However, the disadvantage in productivity is still a problem in programming for accelerators. This paper proposes neoSYCL: a SYCL implementation for SX-Aurora TSUBASA, aiming to improve productivity and achieve comparable performance with native implementations. Unlike other implementations, neoSYCL can identify and separate the kernel part of the SYCL code at the source code level. Thus, this approach can easily be moved to any heterogeneous architectures using the offload programming model. In this paper, we show the evaluation results on SX-Aurora TSUBASA. To quantitatively discuss not only performance but also the productivity, we use two different benchmarks and code-complexity metrics for the evaluation. The results show that neoSYCL can improve productivity while reaching the same performance as native implementations.
\end{abstract}

\section{CCS CONCEPTS}

- Software and its engineering $\rightarrow$ Parallel programming languages.

\section{KEYWORDS}

SYCL, NEC SX-Aurora, Heterogeneous computing, LLVM

\section{ACM Reference Format:}

Yinan Ke, Mulya Agung, and Hiroyuki Takizawa. 2021. neoSYCL: a SYCL implementation for SX-Aurora TSUBASA. In The International Conference on High Performance Computing in Asia-Pacific Region (HPC Asia 2021), January 20-22, 2021, Virtual Event, Republic of Korea. ACM, New York, NY, USA, 8 pages. https://doi.org/10.1145/3432261.3432268

\section{INTRODUCTION}

Today's programmers need to make good use of the system performance, considering its architecture to achieve high performance. In the last few years, the high performance computing (HPC) world has moved to more heterogeneous architectures. It is well-known that a large part of the available computing power of a supercomputer today is provided by various accelerators and co-processors. Therefore, it has become a standard practice to offload a part of application execution to dedicated accelerators. Accelerator vendors

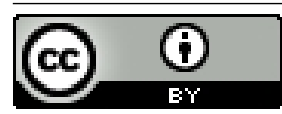

This work is licensed under a Creative Commons Attribution International 4.0 License HPC Asia 2021, January 20-22, 2021, Virtual Event, Republic of Korea (C) 2021 Copyright held by the owner/author(s).

ACM ISBN 978-1-4503-8842-9/21/01

https://doi.org/10.1145/3432261.3432268 also provide offloading mechanisms such as kernel execution and asynchronous data transfer [4].

NEC SX-Aurora TSUBASA (SX-Aurora) is a computing system equipped with a Vector Engine (VE), which is a vector computer on a PCI-Express card [17]. To achieve high sustained performance, a VE provides high memory bandwidth by introducing a new memory integration technology. A single VE provides a memory bandwidth of 0.75 to $1.53 \mathrm{~TB} / \mathrm{s}$, with eight cores and six High Bandwidth Memory 2 (HBM2) modules [15].

NEC provides two different execution models that can be used for SX-Aurora. The most common execution model is native execution on a VE, which avoids the complexity and overhead of memory transfers between the host and VEs. However, native execution often requires a user to compile third-party libraries with the device compiler. It may need the user to modify parts of the source code for various reasons. For example, the device compiler may not support some new features defined in the latest $\mathrm{C}++$ standard. Furthermore, suppose a whole application is executed on a VE. In that case, we need to make the whole application code vector-friendly to achieve high sustained-performance. However, depending on various factors such as the algorithms in the application, it could be hard to optimize the whole code. Considering such a situation, NEC provides the Vector Engine Offloading (VEO) framework [12] to offload only performance-sensitive kernel parts of an application to VEs. As a result, we focus only on optimizing the kernels and making them vector-friendly.

Komatsu et al. have shown that VEs can significantly accelerate memory-intensive vectorizable applications [8]. By offloading a part of an application, we can expect to benefit from the VE performance even if only the part is vector-friendly and the others are not. However, offload programming for the collaboration of different kinds of processors is different from programming only for VEs. Since different kinds of processors may require different programming models, offload programming is likely to be difficult. As shown in Figure 1, we need to write two kinds of codes: host code and device code. The host code runs on the host, while the device code is usually a kernel part of the application and runs on the accelerator, also called the device. We also need to write some glue codes for the collaboration of the host and the device codes. For example, the host code loads the device code at runtime for offloading. Therefore, one difficulty in offload programming is productivity, and will still be a problem for current and future parallel architectures.

SYCL is a single-source programming model that allows us to run the same code on various accelerators or even just on the host. Although there are already some SYCL implementations, currently, there is no SYCL implementation available for SX-Aurora. Besides, most of the implementations assume to use OpenCL for offloading kernel execution to accelerators. There is an OpenCL-like offload 


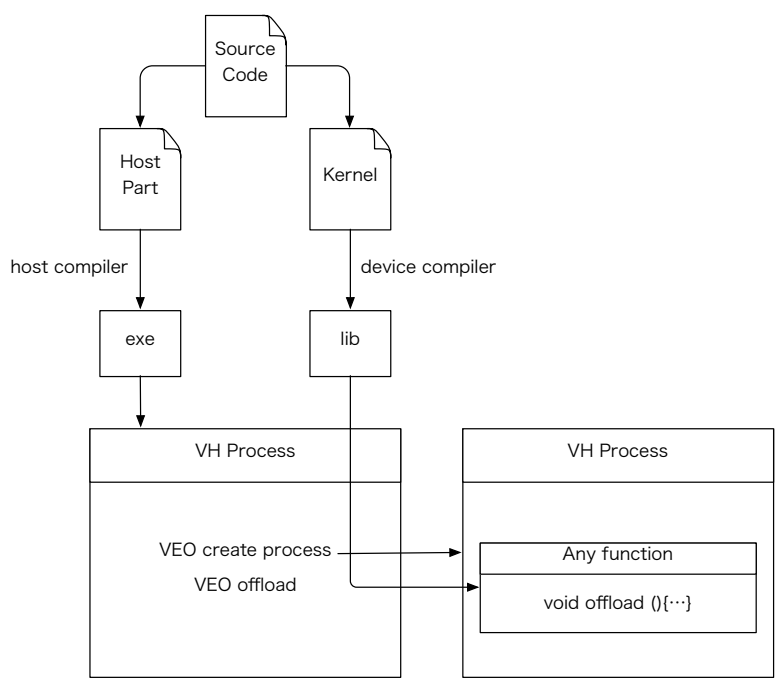

Figure 1: The offloading model in SX-Aurora

programming framework that supports SX-Aurora [14], but it does not provide critical features such as the support of Standard Portable Intermediate Representation (SPIR) format [7], which is required by most SYCL implementations. Under such a situation, we need a stand-alone SYCL implementation that does not assume any specific environments at runtime. It separates kernel parts from the others so that the parts can be compiled with any compilers.

In this paper, we propose a new SYCL implementation supporting offload programming for SX-Aurora. With the proposed SYCL implementation, named neoSYCL, this paper discusses the productivity and performance of SYCL programming for SX-Aurora, in comparison with the native offload programming API.

The main contributions of this paper are summarized as follows.

(1) Present a new approach that separates kernels at the sourcecode level, named kernel outlining. It can easily be moved to any similar heterogeneous architectures.

(2) Provide the first SYCL implementation for SX-Aurora, named neoSYCL, which is a high-level modern $\mathrm{C}++$ template library for single-source programming for multi-core CPUs and SXAurora.

(3) Compare the productivity of neoSYCL with that of VEO by using code complexity metrics.

(4) Demonstrate that neoSYCL can achieve almost the same performance as VEO for two different benchmarks.

\section{RELATED WORK}

In this section, we will give an overview of the techniques related to our proposal.

SYCL (pronounced 'sickle') [3] is a royalty-free, cross-platform abstraction layer that enables code for heterogeneous processors to be written using standard ISO $\mathrm{C}++$ with the host and the kernel codes for an application contained in the same source file.

We give a summary of current open-source SYCL implementations as follows. triSYCL [6] is an open-source implementation based on $\mathrm{C}++17$ and various Boost libraries [13]. triSYCL uses a specific compiler to outline the kernel code. However, different from our approach, the specific compiler generates kernels in the SPIR format. Thus, the device compiler must be able to compile the SPIR codes, which could be a severe requirement when using vendor compilers. hipSYCL [1] is also an open-source implementation based on the modern $\mathrm{C}++$ standard. It builds on the CUDA/HIP Clang frontend, and provides a Clang plugin to support SYCL constructs. However, since the kernel execution is offloaded to CUDA/HIP devices, hipSYCL relies on some features provided by CUDA/HIP drivers. sycl-gtx [18] is another open-source implementation that can be used with any host compiler, built on top of OpenCL 1.2. However, one limitation is that it needs non-standard C preprocessor macros in the control flow, such as SYCL_IF and SYCL_END.

In the case of offload programming on SX-Aurora, there are also some other candidates. The OpenCL-like offload programming framework [14] allows us to migrate any existing OpenCL projects to VEs. Thus, it can express the collaboration between a host and a VE to use both the two different processors. However, this implementation still needs us to divide the source code into the host and kernel parts. In OpenCL, single-source programming can be achieved by using the SPIR format. However, similar to triSYCL, it needs a single compiler that can compile both the host code and the SPIR format. Besides, although an OpenMP implementation for SX-Aurora is also under development [14], the OpenMP approach does not allow an application code to easily use device-specific features nor environments, such as existing libraries for the device.

In contrast to all the related work, this work targets SYCL implementation for SX-Aurora. The implementations proposed by the related work are not applicable to SX-Aurora because they assume using specific devices and compilers, which are not VEs nor NEC's proprietary compiler.

\section{THE PROPOSED SYCL IMPLEMENTATION}

neoSYCL is a high-level modern $\mathrm{C}++$ implementation of the SYCL standard. It is built assuming modern $\mathrm{C}++$ features, such as lambda functions, standard threads, and constant expressions. As with other SYCL implementations, neoSYCL enables users to write source code once and execute on different kinds of devices. The implementation internally uses VEO and std: : thread as programming models for VEs and CPUs, respectively.

\subsection{Queue, Buffer and Accessor}

The basic concepts defined in the SYCL specification [3] are queues, buffers, and accessors. To run some code on a device, we need to create a SYCL queue first. After that, we use the submit function to submit some kernels to the device. The kernels can asynchronously be executed on the device. In the meanwhile, the data containers are represented as SYCL buffers. To access data in the buffer, we need to create a SYCL accessor by calling the get_access function of the SYCL buffer.

In neoSYCL, we can create two kinds of queues, a default queue for CPU execution, and a VE queue for VE execution. Inside those queues, each kernel execution is managed by a dedicated thread created in the $\mathrm{C}++$ standard way. Therefore, the kernel execution in the queue can be done asynchronously. 
Table 1: Numeration of access modes support in neoSYCL

\begin{tabular}{l|l}
\hline Mode & Description \\
\hline access::mode::read & Read-only access. \\
access::mode::write & Write-only access, previous con- \\
& tents not visible. \\
access::mode::read_write & Read and write access. \\
\hline
\end{tabular}

A buffer can be associated with a $1 \mathrm{D}, 2 \mathrm{D}$, or $3 \mathrm{D}$ array that is accessible from kernels by using the corresponding accessor class object. Thus, buffers use a $\mathrm{C}++$ template with two parameters, the type and dimension of the data stored in the buffer. In the neoSYCL implementation, a buffer is implemented as a standard $\mathrm{C}++$ array Therefore, copying data to the VE can be done by copying the whole array to the VE's memory space.

An accessor provides a mechanism to access the data managed by a buffer. The access mode of an accessor that is used to specify how to access the buffer is being provided. This information is used by the neoSYCL runtime to ensure that any data dependencies are resolved by enqueuing any data transfers before or after the execution of a kernel. Also, we do not need to copy the whole buffer back to the host memory for the read-only case, which can significantly improve performance. Table 1 shows the list of access modes that can be set on an accessor. To guarantee the thread-safety of data accesses from different threads and kernels, neoSYCL uses mutexes defined in standard $\mathrm{C}++$. For the read-only buffer, we only need to require the share-mutex instead of the standard mutex. Thus, multiple threads can access one read-only buffer at the same time.

\subsection{Offloading Framework}

Generally, to offload a kernel to a VE, the host code needs to initialize the computing device, sends necessary data to the computing device, and copies the result back after the computation. On one hand, NEC provides an offload library called VEO, which can accept kernels written in standard $\mathrm{C} / \mathrm{C}++$. The neoSYCL implementation uses the VEO library to load and call the VE kernel. On the other hand, we need to vectorize the VE kernel to achieve high performance, which can be done automatically by the NEC compiler. In the neoSYCL implementation, a kernel part is generated as source code. Therefore, the kernel code can be automatically vectorized by the NEC compiler.

\subsection{Kernel Outlining}

In order to fully support the single-source programming defined in SYCL, neoSYCL uses the Clang [9] frontend of LLVM [10] to automatically generate kernel codes. neoSYCL needs to separate the host and kernel codes so that they can be compiled with different compilers. In the case of SX-Aurora, kernels need to be compiled with NEC's proprietary compiler. Therefore, we develop a tool to extract only kernels from a SYCL code. It can analysis the data dependency and gernerate the kernels as $\mathrm{C} / \mathrm{C}++$ functions, so that the neoSYCL runtime can invoke the kernels. As Figure 2 shows, the kernel outlining uses a $\mathrm{C}++$ source file as input, extracts only

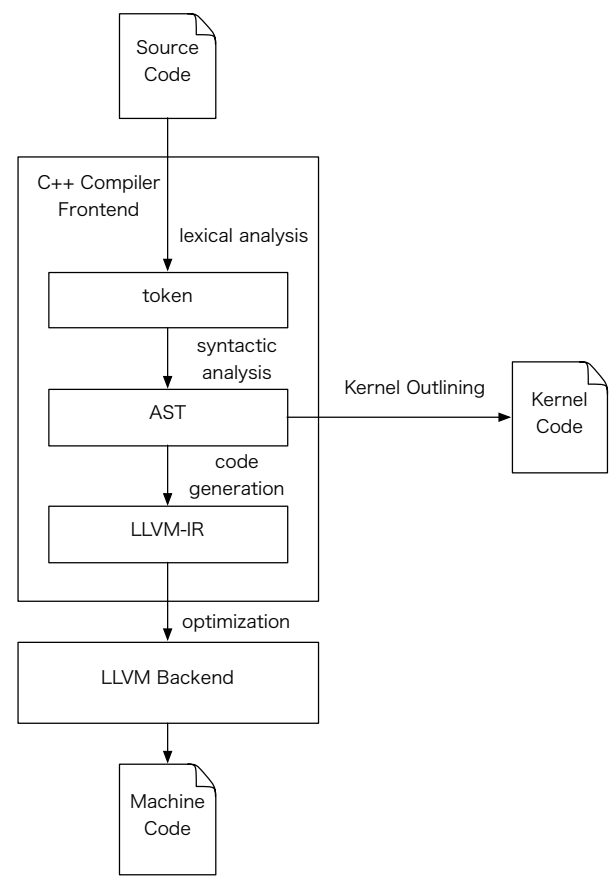

Figure 2: The kernel outlining compiler

the SYCL kernels, and outputs the kernels that can be enqueued from host code by the associated neoSYCL runtime.

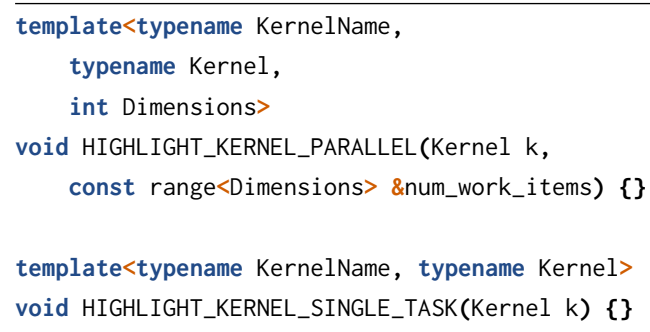

Listing 1. The dummy template functions

In the neoSYCL implementation, after submitting a kernel to the device queue, the execution flow goes into a dummy function, which will be used by the neoSYCL compiler. As shown in Listing 1 , it is implemented as a template function. All of the kernel information, including the data types, dimensions, loop range, and kernel name, are stored in the template arguments. At the compiling step, the neoSYCL compiler can identify that template function from the Clang AST [9] by its name. Therefore, we can get the required information from the AST node, which will be used when generating the kernel code.

After the kernel has been identified from the AST, the last step is to dump the kernel as source code. In LLVM, there are two approaches for the transformation. The first approach is to extract kernels at the LLVM-IR [10] level. Although this approach can use several useful features of LLVM IR, the LLVM IR code must be again converted to $\mathrm{C}$ codes for using the native $\mathrm{C}$ compiler for the 
compilation. The second approach is to extract kernels as strings, i.e., at the source-code level, using the clang: : SourceManager and the clang: : Rewriter class and of Clang.

Since the purpose of this work is to provide the SYCL implementation that can use any compiler for the kernel compilation, this paper uses the latter approach. In the future, we plan to combine the IR approach with current work to take advantage of LLVM IR features.

In the source code level approach, the first step is analyzing the argument dependency. To generate a kernel, we need to know which arguments will be used in the computation. In SYCL specification, all of the data accesses are managed by accessors. In Clang AST, a lambda function is translated into a $\mathrm{C}++$ class, the arguments out of the lambda function body are copied as a reference or copy by value. Thus, by going through the AST context, we can know which accessor has been required, and then get the buffer it belongs to, and also the basic types. Then, we use the pointer of the basic types as the kernel arguments. At runtime, VEO takes the responsibility of data allocation and copy.

The second step is to generate the kernel body. In the SYCL specification, there are two kinds of the kernel: single_task and parallel_for. For single_task, it looks like a simple lambda function, without any parallelism. Thus, we can just dump the function body from the AST node. For the case of parallel_for, the kernel body will be executed many times, an i tem will be passed into as a parameter, which will work similarly as the iterator defined in standard C++. In SYCL standard, it does not define how the kernel execution will be implemented. In the case of neoSYCL, considering the auto-vectorization provided by the NEC compiler, we generate the body of parallel_for kernel as a for-loop. Besides, another possible option is to optimize the kernel body generation with OpenMP. However, since the usage of OpenMP sometimes disables the auto-vectorization feature in the NEC compiler, this version may not achieve satisfying performance. Therefore, this paper does not use OpenMP for work-sharing of the generated for-loop.

After the kernel outlining, the kernels are represented as functions. In the SYCL runtime, we can invoke the kernel by its function name. However, the current $\mathrm{C}++$ standard does not fully support reflection [11]. Thus, we cannot identify the class and function names at runtime. To address this problem, we define a macro that provides the function name information as a $\mathrm{C}++$ string object. Listing 2 shows the definition of the macro. The function name of each kernel is then generated automatically by using this macro in source-to-source transformation.

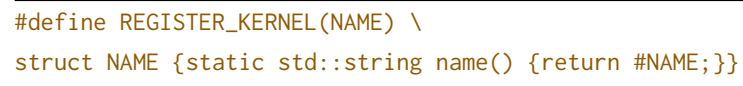

Listing 2. The kernel register macro

To explain how the kernel outlining works, we use an example of SYCL code consisting of two kernel codes, as shown in Listing 3. This code submits two SYCL tasks with parallel_for and single_task executions. After analyzing the argument dependencies, neoSYCL generates two kernel codes. Listings 4 and 5 show the generated kernels for the single_task and parallel_for executions, respectively.
For single_task, the outlining compiler can copy the kernel code out directly from the SYCL code. Meanwhile, for parallel_for, as mentioned earlier, the outlining compiler will generate a for-loop surrounding the function body. As shown in Listing 5, the for-loop size is determined by the arguments $\mathrm{N}$ and STEP.

During compilation, the outlining compiler will write all of the generated kernel codes into one source file. As mentioned in Section 3.2, the source file can be compiled by NEC compiler and generated as a dynamic library. At runtime, when the kernels are submitted to the device queue of SX-Aurora, VEO will load the dynamic library one time. Then, these kernels will be invoked by their function names and executed asynchronously.

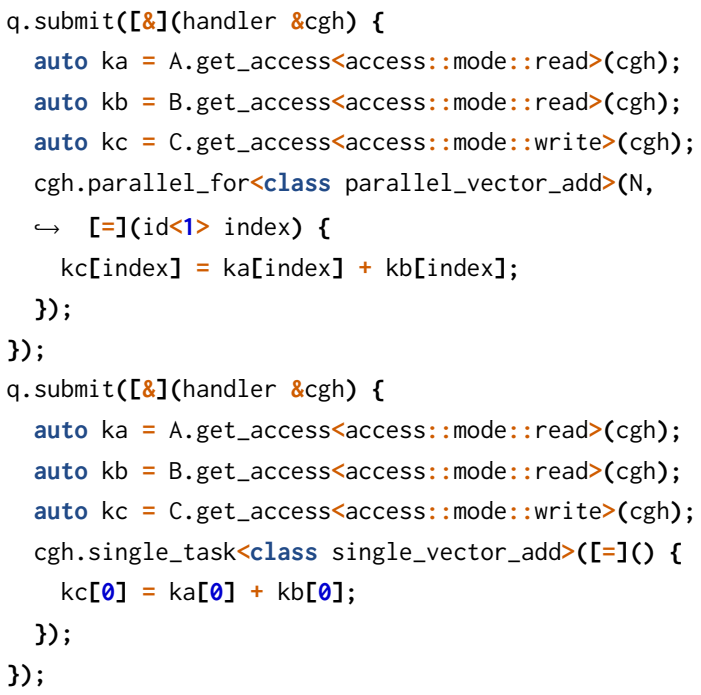

Listing 3. Example of SYCL kernels

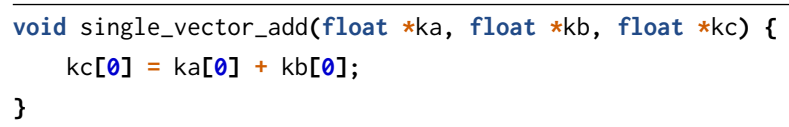

Listing 4. An example of generated kernels (single_task)

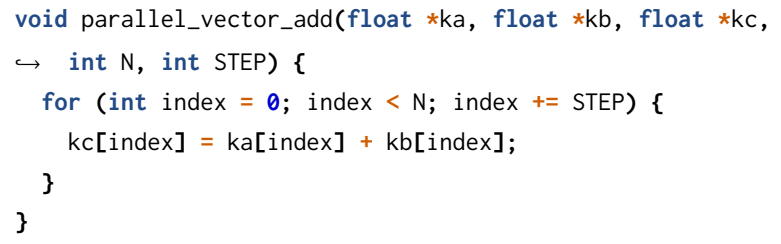

Listing 5. An example of generated kernels (parallel_for)

\subsection{Usage Example}

In order to show how to write code with SYCL, this section shows a simple Triad application from GPU-STREAM benchmark suite [2]. It calculates every element of a vector as the sum of two elements from different arrays, while the second one is scaled by a constant factor. 
A simple sequential implementation of Triad can be presented in Listing 6.

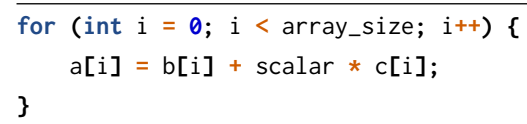

Listing 6. A simple Triad implementation

To implement Triad with neoSYCL, as the first step, we need to wrap each vector with a SYCL buffer and create a device queue. In this case, all of the vectors are represented as 1-D buffers. As shown in Listing 7, we declare three buffers and initialize the value of each element to $0 . T$ is a template argument that represents the data type. The actual data type of $\mathrm{T}$ will be known at the compilation time.

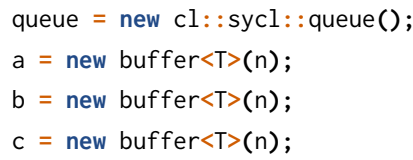

Listing 7. The declaration of queue and buffer

Then we submit a task by calling the submit function defined in the queue. Inside the task, before invoking the kernel computation, we need to create the accessor from the buffer. In the case of Triad, we only need write access for buffer $a$ and read access for buffers $b$ and c. Thus, we only need to copy buffers $b$ and $c$ to device memory and copy back the data of buffer $a$. After that, we can start the kernel computation. The computation of Triad is implemented with the parallel_for strategy. Since we use a compiler plugin to extract kernel code at the compilation time, the kernel code is generated as shown in Listing 9. In the kernel code, ka, kb, and kc are generated from the accessors defined in advance. $\mathrm{N}$ represents the range size of the parallel_for loop.

After the kernel submission, the kernel is executed in an asynchronous way. We wait for the kernel to complete the execution by calling the wait() function. This function is a member function of the queue class.

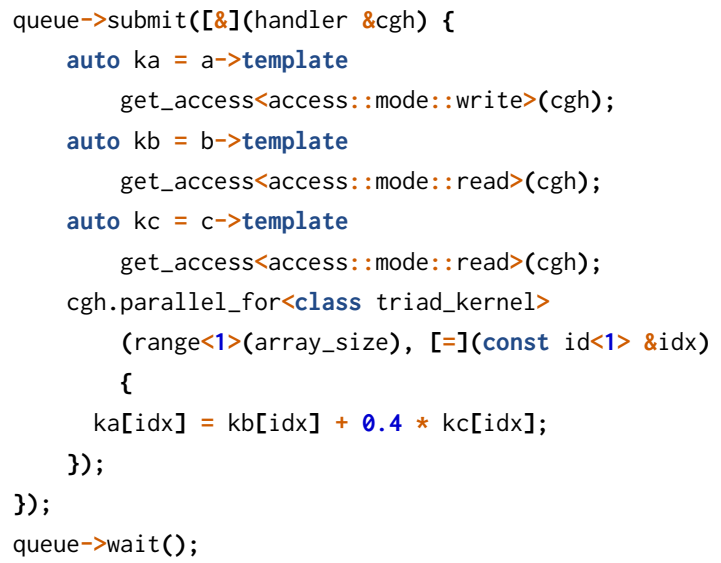

Listing 8. Task submission
Table 2: System specifications

\begin{tabular}{|c|c|}
\hline & NEC SX-Aurora TSUBASA A100-1 \\
\hline VH Processor & Intel(R) Xeon(R) Gold $6126 \mathrm{CPU}$ \\
\hline VH Memory & 96 Gbytes \\
\hline VH Compiler & Clang version 12.0 .0 \\
\hline VE Processor & NEC Vector Engine Type-10C \\
\hline VE Memory & 24 Gbytes \\
\hline VE Compiler & NEC $C++$ compiler 2.5.1 \\
\hline Operating system & CentOS Linux 7.7.1908 \\
\hline \multicolumn{2}{|c|}{$\begin{array}{l}\text { int triad_kernel(double } * k a \text {, double } * k b \text {, double } * k c \text {, int } N \text { ) } \\
\text { for }(\text { int } i=0 ; i<N ; i++)\{ \\
\quad k a[i]=k b[i]+0.4 * k c[i] \\
\text { \} } \\
\text { return } 0 \text {; }\end{array}$} \\
\hline
\end{tabular}

Listing 9. Generated kernel

\section{EVALUATION AND DISCUSSIONS}

In this section, we discuss our evaluation results using two benchmark applications, GPU-STREAM and N-body simulation. GPUSTREAM is often used to measure the sustained memory bandwidth by executing a simple kernel loop. Thus, it can clearly measure the runtime overhead caused by the abstraction, so-called abstraction penalty. On the other hand, N-body is used as an example of SYCL applications that can benefit from offloading kernels to accelerators. In this work, we implement these two benchmarks using neoSYCL and VEO. For each application, we show the performance differences between its VEO and neoSYCL implementations. We compare the performances by measuring the execution times of 100 runs, and calculating the average execution time for each implementation. The specifications of the system used in the following experiments are listed in Table 2.

\subsection{GPU-STREAM}

GPU-STREAM [2] is a benchmark suite which can measure memory transfer rates to/from global device memory on GPUs. By comparing the performance difference with two implementations, neoSYCL and VEO, it can give a clear view of whether there is an overhead with the SYCL runtime and how significant it is.

The results presented in Figure 3 show that the SYCL implementation is $0.42 \%$ slower than the native VEO implementation, with $0.59 \%$ worst slow-down on the dot kernel. Figure 4 shows the bandwidths of two implementations on different array sizes, resulting in $1.6 \%$ slow-down in the worst case. The bandwidth scales linearly down on all implementations due to the increases of the array size. In this case, the SYCL implementation behaves similarly to the VEO implementation. Thus, from the results, we can see that the high-level abstraction of SYCL buffers does not significantly affect the memory access performance. Compare with the native implementation, neoSYCL uses some modern features provided 


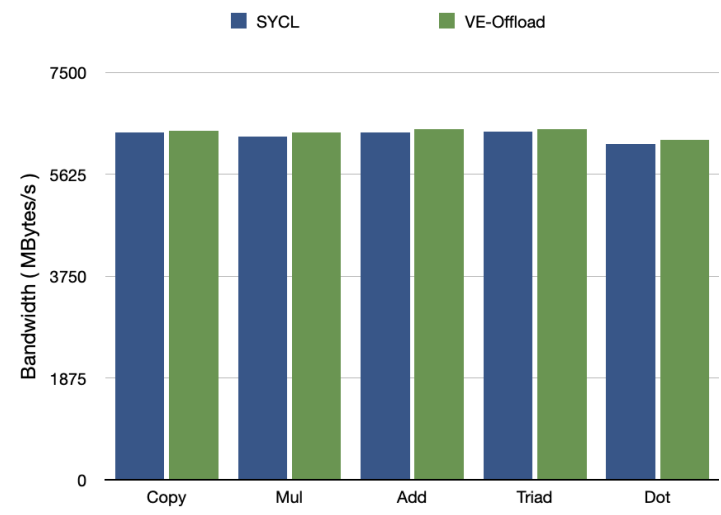

Figure 3: Results of STREAM benchmark with two different implementations

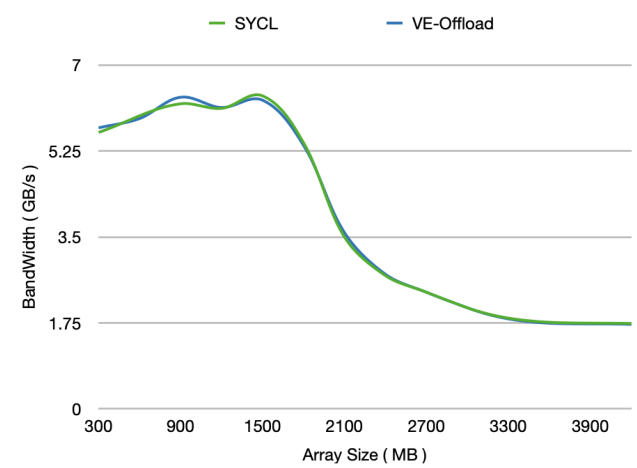

Figure 4: Results of STREAM benchmark scaling from small to larger arrays

in $\mathrm{C}++$ standard. For example, since the kernel execution will be asynchronous, to ensure the data consistency between different kernels, neoSYCL uses some synchronization primitives such as mutex. Thus, the SYCL implementation would have additional overheads.

\section{$4.2 \quad \mathrm{~N}$-body}

The N-body simulation computes the motion of particles that interact with one another through physical forces. In this work, the main parts of the benchmark come from a simple gravitational N-body simulation [5]. The origin code is only implemented targeting at OpenMP and CUDA. Thus we have implemented the SYCL and VEO versions to make it possible to run the code on SX-Aurora in this work. In the case of its naive implementation, the computational complexity is $O\left(N^{2}\right)$, which means the simulation time grows proportionally to the square of the number of particles. As shown in Listing 10, the computation part consists of two loops, which are also the most time-consuming parts. In the neoSYCL and also VEO implementations, only these two parts are offloaded to the VE, and the others are executed on the host.

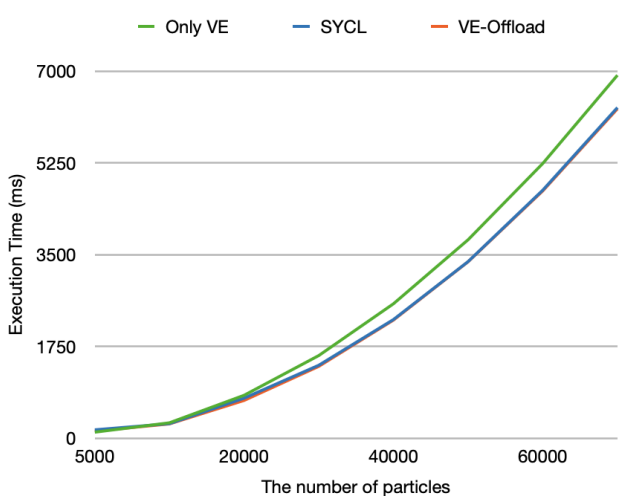

Figure 5: Results of N-body simulation with different input sizes

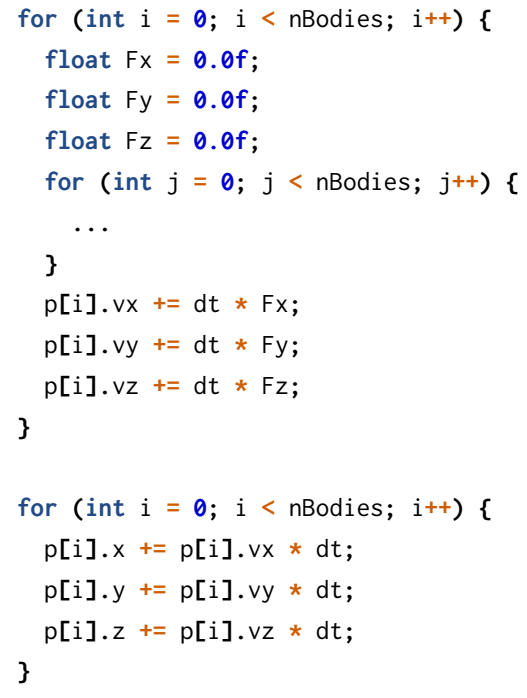

Listing 10. Code Sample of N-Body

In this evaluation, we also implemented another version called VE-only that simply executes the whole simulation on the VE. This implementation does not need to transfer data between the host memory and the device memory. However, VE-only needs to execute not only vector-friendly kernels but also the others on the VE. Therefore, we can discuss the performance benefit from offloading and the cost of data transfers by using the VE-only implementation.

Figure 5 shows the total execution time of each implementation for executing 10 iterations. The input size represents the number of particles in the simulation. For all implementations, the execution time scales linearly with the data size. The SYCL implementation is about $1.7 \%$ slower on average than the VEO implementation. Both the SYCL and VEO implementations perform better than the VE-only implementation, even though the data transfer time is included in their total execution time. As mentioned previously, there are two for-loops in the simulation code. For NEC compiler, only the loop parts can be automatically vectorized. Thus, VEs 


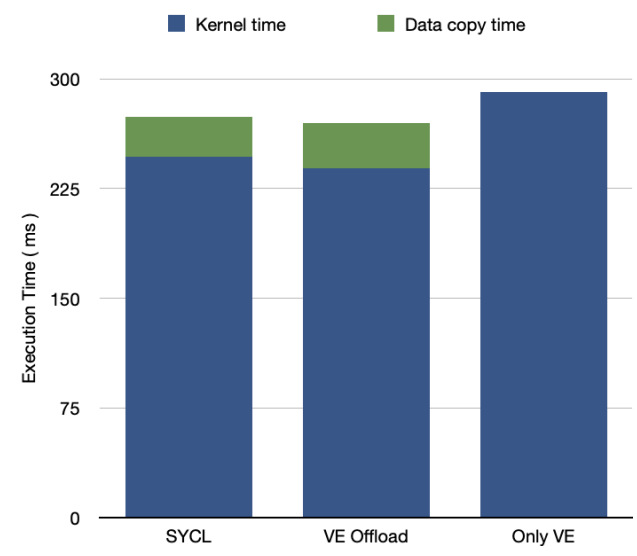

Figure 6: The detail execution time of $\mathrm{N}$-body (10,000 input size)

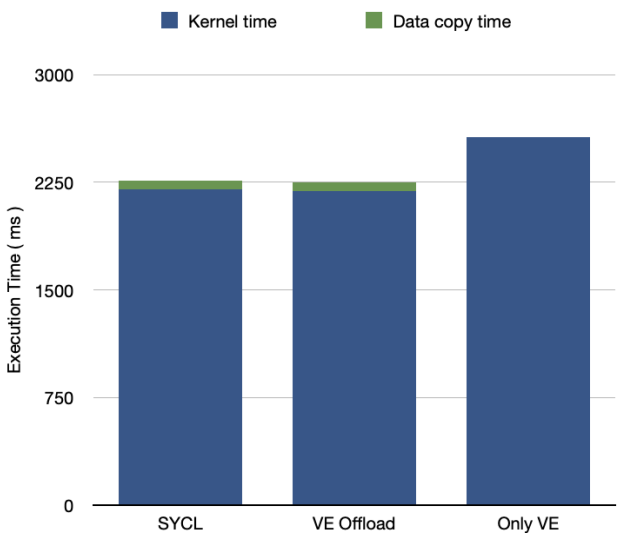

Figure 7: The detail execution time of $\mathrm{N}$-body $(40,000$ input size)

can efficiently work only for these parts. These results also show the benefits of using our method that the device compiler can automatically vectorize the kernel code. Figures 6 and 7 give a clear view of the kernel time and data copy on different implementations. For larger array sizes, the time spent on kernel execution becomes large enough to hide latencies introduced by the offloading.

\subsection{Complexity Metrics}

One important purpose of developing the neoSYCL library is to simplify programming on heterogeneous architectures, thus increasing the productivity of programmers. However, there are several options to show that one application implementation is less complex than another. In this paper, we use a code complexity analyzer, called lizard [16], to measure the code complexities of different implementations. It is chosen because it can measure several metrics of the complexities, and it supports $\mathrm{C}$ and $\mathrm{C}++$, which are used in our evaluation.
Table 3: Metrics Calculated on SYCL and VE-Offload Implementations

\begin{tabular}{c|c|ccc}
\hline Applicaton & VERSION & NLOC & AvgCCN & Avg.token \\
\hline \multirow{2}{*}{ STREAM } & SYCL & 148 & 1.2 & 96 \\
& VE-Offload & 296 & 3.8 & 159.7 \\
\hline \multirow{2}{*}{ N-body } & SYCL & 86 & 2.7 & 233.3 \\
& VE-Offload & 166 & 5 & 240.2 \\
\hline
\end{tabular}

In this section, we compare neoSYCL and VEO implementations of the benchmarks discussed so far in terms of code metrics, including the NLOC (the number of lines of code without comments), $\mathrm{CCN}$ (cyclomatic complexity number) and the Avg.token (average token count of functions). The token count represents the number of distinct operators and distinct operands in a function. Before calculating the metrics, all blank lines and comments are removed to make sure that the results are correct.

Table 3 shows the results in those metrics. It is easy to find that on each metric, neoSYCL is better than VEO. In the case of STREAM, neoSYCL is at least two times better than VEO in both NLOC and AvgCCN. In the case of N-body, neoSYCL shows better results in both NLOC and AvgCCN. However, they show almost the same Avg.token score. This is because the token count of the data initialization part is higher than that of the kernel part, which is executed on the host side and cannot benefit from neoSYCL. In conclusion, neoSYCL abstraction mechanisms significantly decrease the resultant code complexity, compared with the native implementation.

\section{CONCLUSIONS AND FUTURE WORK}

In this paper, we have presented neoSYCL: a SYCL implementation available for SX-Aurora. neoSYCL can automatically offload parts of application execution to VEs, by generating the kernel code at the compilation time. Our performance evaluation clearly demonstrates that neoSYCL can achieve comparable performance with the native offloading library provided by NEC. In addition, neoSYCL can also improve programming productivity significantly. Accordingly, these results clearly show that neoSYCL can help programmers obtain a reasonable performance with lower effort.

The current neoSYCL implementation already supports most of major features in the SYCL standard. However, SYCL is originally based on OpenCL, and neoSYCL currently does not support some of features that need OpenCL-specific features, such as sycl: : image. Hence, we are planning to further improve the neoSYCL implementation to fully support the SYCL specification. Since neoSYCL can extract the kernel code from the SYCL application automatically, it is also applicable to any SYCL applications developed for different systems. In the near future, we will extend our method for other devices, such as NVIDIA and AMD GPUs, and also FPGAs.

\section{ACKNOWLEDGMENTS}

This work partially supported by MEXT Next Generation HighPerformance Computing Infrastructures and Applications R\&D Program "R\&D of A Quantum-Annealing-Assisted Next Generation 
HPC Infrastructure and its Applications" Grant-in-Aid for Scientific Research (A) \#20H00593.

\section{REFERENCES}

[1] Aksel Alpay. 2019. hipSYCL. https://github.com/illuhad/hipSYCL

[2] Tom Deakin, James Price, Matt Martineau, and Simon McIntosh-Smith. 2016. GPU-STREAM v2. 0: Benchmarking the achievable memory bandwidth of manycore processors across diverse parallel programming models. In International Conference on High Performance Computing. Springer, 489-507.

[3] Khronos SYCL Working Group. 2019. The SYCL 1.2. 1 Specification.

[4] Mark Harris. 2012. How to overlap data transfers in CUDA C/C++ URL http://devblogs. nvidia. com/parallelforall/how-overlap-data-transfers-cuda-cc (2012).

[5] Mark Harris. 2014. mini-nbody: A simple N-body Code. Retrieved March 26 (2014), 2019.

[6] Ronan Keryell. 2014. triSYCL. https://github.com/triSYCL/triSYCL.

[7] John Kessenich, Boaz Ouriel, and Raun Krisch. 2018. SPIR-V Specification Khronos Group 3 (2018).

[8] Kazuhiko Komatsu, Shintaro Momose, Yoko Isobe, Osamu Watanabe, Akihiro Musa, Mitsuo Yokokawa, Toshikazu Aoyama, Masayuki Sato, and Hiroaki Kobayashi. 2018. Performance evaluation of a vector supercomputer SX-Aurora TSUBASA. In SC18: International Conference for High Performance Computing, Networking, Storage and Analysis. IEEE, 685-696.
[9] Chris Lattner. 2008. LLVM and Clang: Next generation compiler technology. In The BSD conference, Vol. 5.

[10] Chris Lattner and Vikram Adve. 2004. LLVM: A compilation framework for lifelong program analysis \& transformation. In International Symposium on Code Generation and Optimization, 2004. CGO 2004. IEEE, 75-86.

[11] Duraid Madina and Russell K Standish. 2001. A system for reflection in C++. Proceedings of AUUG2001: Always on and Everywhere (2001), 207.

[12] Matthias Noack, Erich Focht, and Thomas Steinke. 2019. Heterogeneous active messages for offloading on the NEC SX-Aurora TSUBASA. In 2019 IEEE International Parallel and Distributed Processing Symposium Workshops (IPDPSW). IEEE, 26-35.

[13] Boris Schäling. 2011. The boost $C++$ libraries. Boris Schäling.

[14] Hiroyuki Takizawa, Shinji Shiotsuki, Naoki Ebata, and Ryusuke Egawa. 2019. An OpenCL-Like Offload Programming Framework for SX-Aurora TSUBASA. In 2019 20th International Conference on Parallel and Distributed Computing, Applications and Technologies (PDCAT). IEEE, 282-288.

[15] Yohei Yamada and Shintaro Momose. 2018. Vector engine processor of NEC's brand-new supercomputer SX-Aurora TSUBASA. In Proceedings of A Symposium on High Performance Chips, Hot Chips, Vol. 30. 19-21.

[16] Terry Yin. 2020. lizard. https://github.com/terryyin/lizard.

[17] Mitsuo Yokokawa, Ayano Nakai, Kazuhiko Komatsu, Yuta Watanabe, Yasuhisa Masaoka, Yoko Isobe, and Hiroaki Kobayashi. 2020. I/o performance of the sx-aurora tsubasa. In 2020 IEEE International Parallel and Distributed Processing Symposium Workshops (IPDPSW). IEEE, 27-35.

[18] Peter Žužek. 2019. sycl-gtx. https://github.com/ProGTX/sycl-gtx. 